\title{
A method for scintillation characterization using geodetic receivers operating at $1 \mathrm{~Hz}$
}

\author{
J. M. Juan ${ }^{1}$ - A. Aragon-Angel ${ }^{2}$. \\ J. Sanz ${ }^{1}$ - G. González-Casado ${ }^{1}$. \\ A. Rovira-Garcia ${ }^{1}$
}

Received: October 7, 2016

Accepted: April 19, 2017

\begin{abstract}
Ionospheric scintillation produces strong disruptive effects on Global Navigation Satellite System (GNSS) signals, ranging from degrading performances to rendering these signals useless for accurate navigation. The current paper presents a novel approach to detect scintillation on the GNSS signals based on its effect on the ionospheric-free combination of carrier-phases, i.e. the standard combination of measurements used in Precise Point Positioning (PPP). The method is implemented using actual data, thereby having both its feasibility and its usefulness assessed at the same time. The results identify the main effects of scintillation, which consist of an increased level of noise in the ionosphericfree combination of measurements and the introduction of cycle-slips into the signals. Also discussed is how misdetected cycle-slips contaminate the Rate Of change of the TEC Index (ROTI) values, which is especially important for low-latitude receivers. By considering the effect of single jumps in the individual frequencies, the
\end{abstract}

This work was supported in part by the Spanish Ministry of Science and Innovation project CGL2015-66410-P and in part by the European Space Agency project SCIONAV, contract No. $4000115300 / 15 / \mathrm{NL} / \mathrm{AF}$.

\section{J.M. Juan}

E-mail: jose.miguel.juan@upc.edu

$1 \cdot$ Research Group of Astronomy and Geomatics (gAGE)

Technical University of Catalonia (UPC)

Barcelona, Spain

$2 \cdot$ European Commission, Joint Research Centre (JRC)

Directorate for Space, Security and Migration

Ispra, Italy proposed method is able to isolate, over the combined signal, the frequency experiencing the cycle-slip. Moreover, because of the use of the ionospheric-free combination, the method captures the diffractive nature of the scintillation phenomena that, in the end, is the relevant effect on PPP. Finally, a new scintillation index is introduced that is associated with the degradation of the performance in navigation.

Keywords - Scintillation - ionospheric irregularities · Global Navigation Satellite System (GNSS) · High Accuracy Navigation · cycle-slip detection · scintillation indices

\section{Introduction}

Ionospheric scintillation is related to fluctuations in the intensity and phase of radio signals, including those of the Global Navigation Satellite System (GNSS). The signals are scattered and/or diffracted by irregularities in the electron distribution encountered during travel along the ray propagation path, thereby disrupting the performance of space-based communication and navigation systems. Scintillation causes the GNSS signals to have an increased level of noise or even experience a loss of lock on the receiver tracking. For that reason, scintillation has become one of the major concerns in navigation ( $\mathrm{Pi}$ et al, 2014); therefore, the correction and/or mitigation of the effects of scintillation is one of the current challenges in achieving precise GNSS navigation (see Béniguel et al, 2009). 
The climatology of L-band scintillations has been characterized in several studies involving GNSS receiver networks (e.g. Cervera and Thomas, 2006; Paznukhov et al, 2012); from these studies, it is possible to state that the occurrence of ionospheric scintillation and its intensity are strongly dependent on latitude, local time, season of the year, Solar Cycle, and magnetic activity, presenting a strong degree of day to day variability (Aarons, 1982, 1993). Although scintillation may be a global phenomenon, the occurrence of scintillation is generally clustered around the geomagnetic equator $\left(\phi_{\text {magnetic }}<|25|^{\circ}\right)$ and in both polar regions $\left(\phi_{\text {magnetic }}>\right.$ $\left.|60|^{\circ}\right)$. In the particular case of high latitudes, scintillation appears to be associated with geomagnetic storms or solar events, whereas in equatorial latitudes, it is associated with plasma movements that typically occur after the local sunset (Béniguel et al, 2009).

Concerning the size of the irregularities in the electron distribution, these irregularities can range from metres to tens of kilometres, and their drift velocities (in combination with the scan velocity of the GNSS) can span from tens to hundreds of metres per second, in low latitudes, up to some kilometres per second, in high latitudes (Basu and Basu, 1989). Large-scale variations in the electron density cause changes in the signal refraction; moreover, irregularities up to scale lengths of approximately some hundreds of metres also scatter the signal, causing diffractive effects (Kintner et al, 2009) that can be observed as fading of the signal amplitude. In both cases, the GNSS signals experience rapid phase variations, which can be tracked by specific GNSS receivers that work at higher frequency (typically $50 \mathrm{~Hz}$ ) than standard GNSS receivers. From the data collected from these particular Ionospheric Scintillation Monitoring Receivers (ISMRs), several indices have been developed to measure the effect of scintillation activity on GNSS signals:

The amplitude scintillation index, $S_{4}$ is defined as the standard deviation of the signal power normalized by its mean (Briggs and Parkin, 1963):

$S_{4}=\sqrt{\frac{\left\langle S I^{2}\right\rangle-\langle S I\rangle^{2}}{\langle S I\rangle^{2}}}$

where SI denotes the signal intensity. $S_{4}$ is typically computed over a period of one minute, and its values typically range from 0 to 1 (although it may be greater than 1).
The phase scintillation index, $\sigma_{\varphi}$ is defined as the standard deviation of the de-trended carrier phase (Yeh and Chao-Han, 1982):

$\sigma_{\varphi}=\sqrt{\left\langle\varphi^{2}\right\rangle-\langle\varphi\rangle^{2}}$

where $\varphi$ denotes the de-trended carrier phase measurement. This standard deviation is calculated over a certain time period, typically one minute, and accounts for carrier phase fluctuations. Unlike amplitude fading, these phase fluctuations can also appear in large-scale irregularities (Rino, 1979).

The computation of $\sigma_{\varphi}$ takes advantage of the highfrequency nature of the carrier phase fluctuations associated with scintillation. In this sense, the isolation of the scintillation effect on the carrier phase is performed by filtering the carrier phase, typically with a $6^{\text {th }}$ order high-pass Butterworth filter (Van Dierendonck and Arbesser-Ratsburg, 2004). Thus, all the fluctuations introduced by other effects (such as geometry and tropospheric delay) that usually are of lower frequencies are removed. However, other high-frequency effects that could be present on the carrier phase data, such as the receiver clock errors or cycle-slips, may distort the calculation of the $\sigma_{\varphi}$ index (Humphreys et al, 2005). Moreover, the particular characteristics of the filter can have an influence on the $\sigma_{\varphi}$ retrievals (Forte, 2005).

The common characteristic of the aforementioned scintillation indices is that they rely on high data sampling rates, which for the ISMRs are usually $50 \mathrm{~Hz}$. However, ISMRs are not yet very numerous, and the storage of data at such sampling rate requires substantial memory capabilities. As a consequence, scintillation data are not typically openly available. In this context, scintillation indices computed with data collected at a lower frequency are of great interest.

The Rate Of change of the TEC Index (ROTI) defined in $\mathrm{Pi}$ et al (1997) is an example of a scintillation indicator that can be computed from dual-frequency measurements at low frequency (typically $1 / 30 \mathrm{~Hz}$ ). The ROTI is defined as the standard deviation of the time derivative of the ionospheric delay, i.e. the Total Electron Content (TEC). The Slant TEC (STEC) is measured directly from the geometric-free combination of the carrier phase measurements $\left(L_{G F}=L_{1}-L_{2}\right)$, where the hardware biases and ambiguities of the carrier phases are removed in the derivation:

$R O T I=\frac{1}{M(\epsilon)} \times \sqrt{\left\langle\left(\frac{\Delta L_{G F}}{\Delta t}\right)^{2}\right\rangle-\left\langle\frac{\Delta L_{G F}}{\Delta t}\right\rangle^{2}}$ 
$\mathrm{M}(\epsilon)$ being an obliquity factor, which depends on the satellite elevation $(\epsilon)$, used to mitigate large ROTI values at low elevations.

This standard deviation is computed over a time window of pre-defined length, which depends on the sampling rate (typically 5 minutes with $1 / 30 \mathrm{~Hz}$ data or 1 minute with $1 \mathrm{~Hz}$ data). In fact, ROTI determinations are influenced by the selected sampling rate and time interval for the calculation (Jacobsen, 2014). In addition, a key element in the ROTI computation is the correct identification of the satellite arcs, which depends on the receiver processing algorithms. Moreover, no straightforward relationship exists between the ROTI and the aforementioned $S_{4}$ or $\sigma_{\varphi}$ indices, and their inter-comparisons are usually conducted using statistics (e.g Carrano and Groves, 2007).

Despite these previous drawbacks, the ROTI has become a standard scintillation indicator because of the availability of a large number of receivers operating at lower frequencies (e.g. $1 / 30 \mathrm{~Hz}$ or $1 \mathrm{~Hz}$ ) distributed worldwide and openly accessible (e.g. hundreds of them from the International GNSS Service (IGS), see Beutler et al (1999)). This is not the case of the ISMRs.

Another ionospheric activity indicator suitable for identification of the ionospheric disturbed periods is the Along Arc TEC Rate (AATR) index (Sanz et al, 2014). As in the case of the ROTI, the AATR is also based on the time derivative of the $L_{G F}$ combination; however, the goal of the AATR is to identify the periods and regions with high ionospheric activity (at low or high frequency). In this sense, AATR is sensitive not only to scintillation but also to other ionospheric phenomena that have large temporal or spatial gradients (such as bubbles and ionospheric storms); such phenomena commonly appear jointly with scintillation. Thus, the AATR is a useful parameter to select the periods and regions for which scintillation episodes are expected.

In this paper, a new methodology is presented for detecting scintillation on the GNSS signals with geodetic receivers at $1 \mathrm{~Hz}$ data. The proposed method uses the ionospheric-free combination of carrier phase measurements $\left(L_{I F}\right)$, which is the usual combination in the standard Precise Point Positioning (PPP) (Zumberge et al, 1997). A new scintillation index is defined (ionospheric-free standard deviation or $\sigma_{I F}$ ) that measures the scintillation effect on the $L_{I F}$ combination and, therefore, this index measures the direct effect of scintillation on positioning.
The paper is structured as follows. Sect. 2 describes the data set that is used to introduce the methodology. Sect. 3 provides the details of the method. In Sect. 4, $\sigma_{I F}$ is introduced as a new scintillation indicator that is specifically tailored for navigation applications. Sect. 5 presents comparisons of the new index $\sigma_{I F}$ versus commonly used scintillation indices. Finally, in Sect. 6, the main conclusions of this work are highlighted and summarized.

\section{Experimental data}

The proposed methodology is illustrated using the data gathered by the IGS receiver SEY1 (located in Seychelles Islands), which corresponds to a low latitude during the Day Of Year (DOY) 058 in 2014 (February $\left.27^{t h}, 2014\right)$. The selection of this particular day is two-fold: first, because the date is close to the March Equinox, scintillation episodes are expected to occur in low latitudes at local sunset hours; second, during the last part of DOY 058, an ionospheric storm was triggered by the arrival of charged particles to the Earth that were ejected from the Sun some days before (Sanz et al, 2014), see the $D_{s t}$ index in the bottom plot of Fig. 1.

Such ionospheric disturbances can be monitored by means of the ROTI or AATR index. The AATR is depicted in Fig. 1 for several consecutive days, including the day of interest. Four additional IGS receivers are included in the figure for inter-comparison purposes: another one in low latitude, CHPI (East coast of Brazil); two in high-latitude regions, YELL (Canada, close to the North geomagnetic pole) and MCM4 (Antarctic continent, close to the South geomagnetic pole); and one in a mid-latitude region, USN3 (East coast of the United States of America). The coordinates and model of each respective receiver are provided in Table 1 along with corresponding geomagnetic latitude, the largest AATR value at each location achieved during DOY 058 and the $99^{t h}$ percentile during the whole year 2014, for comparison.

Figure 1 shows that the mid-latitude receiver USN3 presents moderate AATR values during all the week. The largest AATR values correspond to the end of DOY 058 for the high-latitude receivers MCM4 and YELL. These observed AATR values are among the largest values that occurred during 2014 and are associated with the aforementioned ionospheric storm (note that, for these two high latitude receivers, the AATR values dur- 

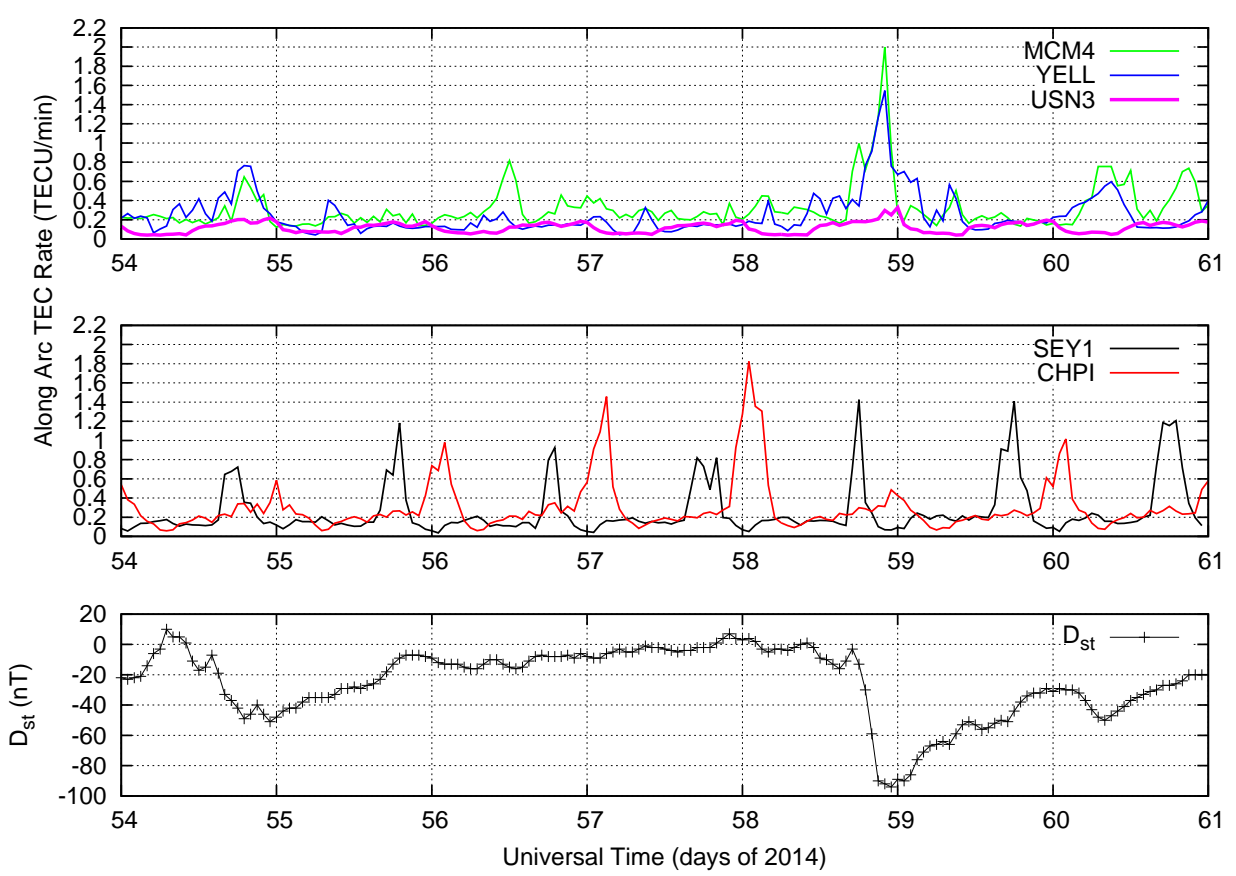

Fig. 1: Ionospheric Activity Indicators from DOY 054 to 061 in 2014 . The top and middle plot depict AATR values at several IGS stations: high latitude: MCM4 (green) and YELL (blue); mid latitude: USN3 (pink); low latitude: SEY1 (black), CHPI (red). The bottom plot depicts the $D_{s t}$ values for the period of study using a black line with pluses. DOY 058 presents the highest values for the AATR and the lowest $D_{s t}$ indices during the period.

Table 1: For selected IGS stations, a detailed list of the receiver type, geographic coordinates, geomagnetic latitude, highest AATR value achieved during DOY 058 and $99^{\text {th }}$ percentile AATR value for year 2014.

\begin{tabular}{llrcccc}
\hline \multirow{2}{*}{ Station } & \multirow{2}{*}{ Receiver type } & \multicolumn{2}{c}{ Coordinates (degrees) } & \multicolumn{2}{c}{ AATR (TECUs/min) } \\
Name & Brand / model & \multicolumn{2}{c}{$\begin{array}{c}\text { Geographic } \\
\text { Lon }\end{array}$} & Lat & Geomagnetic & \multicolumn{2}{c}{$\begin{array}{c}\text { DOY 058 } \\
\text { Max }\end{array}$} & $\begin{array}{c}\text { Year 2014 } \\
99^{t h}\end{array}$ \\
\hline CHPI & JAVAD TRE_G3TH & -45.0 & -22.6 & -12.4 & 1.82 & 0.91 \\
MCM4 & ASHTECH UZ-12 & 166.7 & -77.8 & -78.7 & 2.00 & 0.87 \\
SEY1 & JAVAD TRE_G3TH & 55.5 & -4.6 & -11.2 & 1.42 & 0.94 \\
USN3 & ASHTECH Z-XII3T & -77.1 & 38.7 & 49.9 & 0.30 & 0.19 \\
YELL & JAVAD TRE_G3T & -114.5 & 62.3 & 69.1 & 1.55 & 0.68 \\
\hline
\end{tabular}

ing the rest of the week remain quite moderate). Moreover, the largest AATR values for the two low-latitude receivers (SEY1 and CHPI) are found to occur shortly after the local sunset hours on a daily basis. Examining Table 1, one can also notice that the AATR values for these low latitude receivers, during day 058, correspond to one of the largest values during the whole year 2014 , as was the case for the middle and high latitude stations.

The information extracted from the AATR values regarding the ionospheric activity can be complemented by examining the high ROTI values associated with the data from some satellites for the same receivers in Table 1. This examination is accomplished in Fig. 2, where the ROTI values for four satellite-receiver pairs are depicted. Because we use a sampling rate of $1 \mathrm{~Hz}$ for computing the ROTI, the represented values are higher than the typical values obtained at $1 / 30 \mathrm{~Hz}$ (see Jacobsen, 2014). In any case, from these ROTI values, it can be assumed that the data were gathered under strong scintillation conditions. The ROTI values for the same satellite-receiver pairs are depicted for the previous day in the left plot of Fig. 2. It can be observed that the lowlatitude receivers (SEY1 and CHPI) are also affected by strong scintillation (related with the local sunset), but for the high-latitude receivers (YELL and MCM4) the ROTI values are moderate, because the geomagnetic storm has not started yet. 

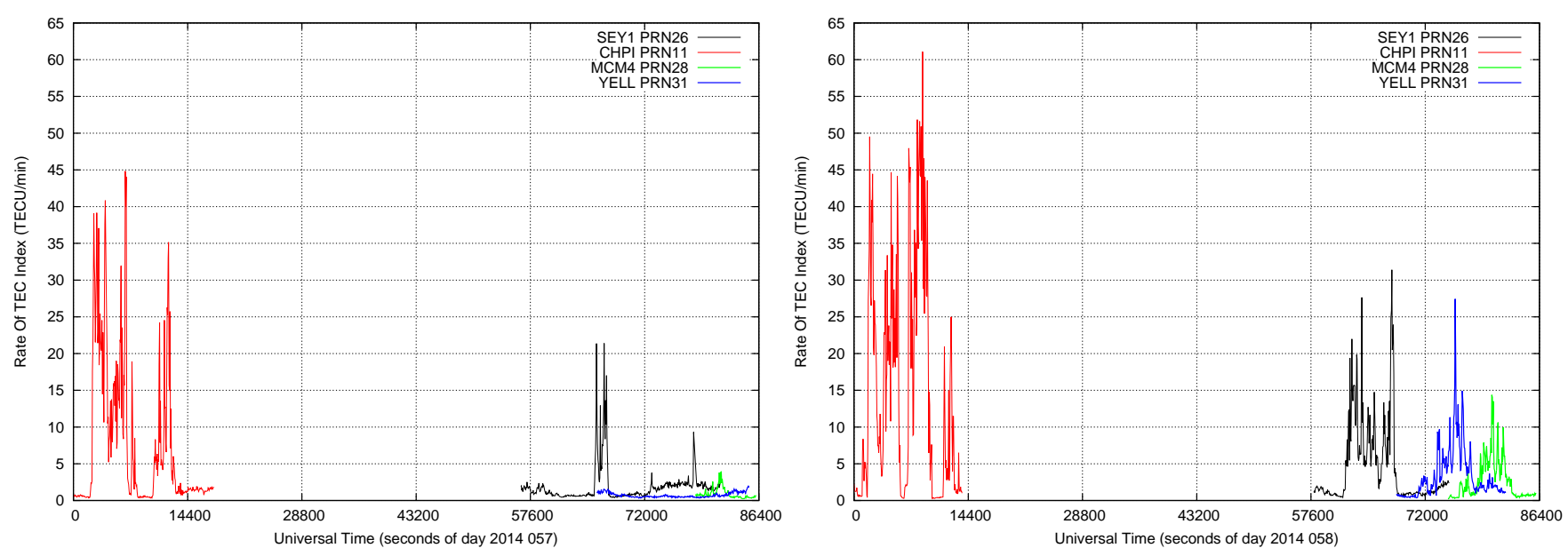

Fig. 2: ROTI values corresponding to DOY 57 (left) and DOY 58 (right) in 2014 at the IGS stations of SEY1 (black), CHPI (red), MCM4 (green) and YELL (blue) for some particular satellites (PRN26, PRN11, PRN28 and PRN31).

\section{Method}

The method proposed in this work is based on using the ionospheric free combination of carrier phase measurements, $L_{I F}$, in metres, defined as:

$L_{I F}=\alpha \cdot \varphi_{1}-\beta \cdot \varphi_{2}$

where $\varphi_{1}$ and $\varphi_{2}$ are the raw carrier phases (expressed in cycles) in the $L_{1}$ and $L_{2}$ GPS frequencies, and the coefficients $\alpha=0.484$ metre/cycle and $\beta=0.377$ metre/cycle are the corresponding conversion factors. In fact, $\alpha$ and $\beta$ represent the variation that the $L_{I F}$ combination experiences after one cycle increment in $L_{1}$ or $L_{2}$, respectively. Note that these figures differ by a factor of $2 \pi$ from those in Carrano et al (2013). If the value of $L_{I F}$ is computed from the $L_{1}$ and $L_{5}$, the corresponding values are $\alpha=0.430$ metre/cycle and $\beta=0.321$ metre/cycle.

The refractive part of the ionospheric delay is wellknown (Crawford, 1968) to depend on $1 / f^{2}$ and can be removed (typically up to a $99.9 \%$ ) with the $L_{I F}$ combination. As a result, $L_{I F}$ is the most common combination used in high-accuracy navigation. Indeed, in nominal conditions, when all the effects on the GNSS signal are taken into account, this combination can be accurately modelled up to the centimetre level (Sanz et al, 2013). Therefore, any non-modelled effect with an impact on the $L_{I F}$ combination greater than few centimetres (as the non-refractive part of the ionospheric scintillation) should be observed.

The methodology is essentially the same than in the standard PPP processing, with some differences that will be highlighted in the next paragraphs. We will illustrate this by considering one of the cases in Fig. 2; specifically, the arc of data from the IGS receiver SEY1 and PRN26.

First, the large cycle-slips that usually occur under perturbed ionospheric activity periods must be identified. Generally, these large cycle-slips cause a loss of lock of the signal. For this purpose, an initial (rough) cycle-slip detector is established, which is similar to the cycle-slip detector described in (Sanz et al, 2013). In the first step, the algorithm uses the Melbourne Wübbena (MW) combination, as in the Turboedit algorithm (Blewitt, 1990). The second step of the cycle-slip detector consists of a polynomial fitting of the last $L_{G F}$ data to predict the next data value (notice that the Turboedit algorithm uses the pseudorange for this purpose). In this second step, a cycle-slip is declared when the actual $L_{G F}$ and the predicted value differ more than 40 centimetres. Notice that, according to the definition of $L_{G F}$, this threshold corresponds to more than one cycleslip on $L_{1}$ or $L_{2}$ in standard PPP this threshold is usually set to a much lower value).

The situation is illustrated for receiver SEY1 and PRN26 in Fig. 3, together with the ionospheric activity sampled by means of the ROTI index (solid line). In this plot, the original $L_{G F}$, which is directly computed from the $L_{1}$ and $L_{2}$ measurements present in the RINEX file, is depicted with red pluses. The blue crosses represent the $L_{G F}$ corresponding to the different continuous arcs of data after the rough cycle-slip detection. Because the carrier phase is aligned with the code after each cycle- 


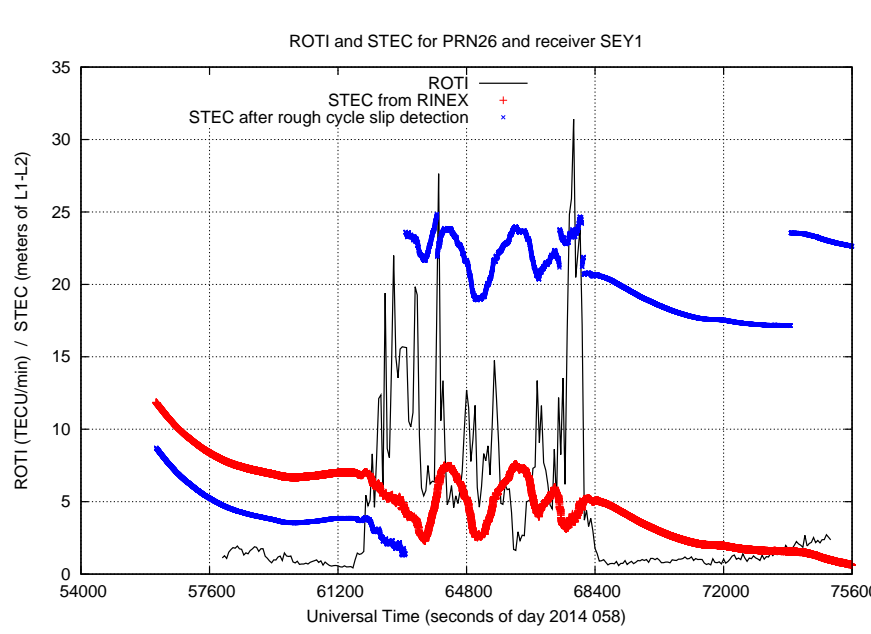

Fig. 3: For IGS receiver SEY1 and PRN26: red pluses, STEC from RINEX without cycle-slip detection; blue crosses, STEC during different continuous arcs of data after rough polynomial-based cycle-slip detection; solid black line, ROTI.

slip, the carrier phase jumps (some of them detected with the MW combination) are enhanced after using this initial cycle-slip detector. Therefore, the continuous arcs can be easily identified.

After this rough detection of the cycle-slips, our efforts are devoted to the study of smaller cycle-slips, which consist of one cycle in either $L_{1}$ or $L_{2}$.

Several authors (e.g. Xu and Morton, 2015) have reported the advantage of using the receiver position and the satellite orbits as part of the de-trending to the GNSS measurements. Similarly, we start from the precise coordinates of the receiver. Going beyond this idea, the second step consists of modelling (as accurately as possible) the physical effects on the $L_{I F}$ combination. These physical effects include tropospheric delays and satellite orbits and clock offsets, as in PPP. Tropospheric delays can be corrected using the IGS tropospheric corrections available at International GNSS Service Products (2014) that are computed for a large number of IGS receivers. Notice that as stated in the IGS website, these corrections have an accuracy of 4 $\mathrm{mm}$ in the zenith direction. Therefore, residuals errors in the tropospheric corrections will be at the level of 1 $\mathrm{cm}$ and easily distinguishable from scintillation effects because varies slowly in time.

Satellite clock offsets are interpolated from precise clock files from the IGS final combined solution; these files are stored at sampling interval of $30 \mathrm{~s}$ and must be interpolated to the required $1 \mathrm{~s}$ cadence of the geode- tic receivers. The final modelling shall be accurate to the centimetre level. At this point, note that the detrending is sensitive to any non-linear behavior of the satellite clock; which could affect the GNSS signal in a similar way than scintillation, as later detailed in Sect. 4. Therefore, it is interesting to include in the study some additional receivers that are not affected by scintillation. With the help of the data from such receivers, eventual anomalies of the satellite clocks can be detected. This is the rationale for the inclusion of the mid-latitude receiver "USN3" in the analysis presented in Sect. 4.

After the de-trending of the ionospheric-free combination, only the receiver clock offset, the carrier phase ambiguities, and scintillation effects should remain as residuals. The outcome of this process is shown in Fig. 4 where the residuals of the $L_{I F}$ are depicted for several satellites in the same plot. Except for a constant per arc associated with the carrier phase ambiguities, all the residuals are found to share a common pattern related with the receiver clock offset. In fact, these are the same terms as in PPP, except for the receiver coordinates (that in the method are known). In this way, these residuals can be described by:

$\Delta L_{I F}^{j}=c \cdot T_{r c v}+B_{I F}^{j}$

where $\Delta L_{I F}^{j}$ is the residual of the $L_{I F}$ combination for satellite " $\mathrm{j}$ ", "c" is the speed of light, $T_{r c v}$ is the receiver clock offset, and $B_{I F}^{j}$ is the carrier phase ambiguity.

At this point, since the clock offset of this receiver does not present large variations, some cycle-slips can be easily identified (for example in the PRN02 residuals).

Starting from the residuals depicted in Fig. 4, in a third step, the carrier-phase ambiguities (per arc) and the receiver clock offset (per epoch) are estimated using Eq.(5). This assumes that not all the satellites are affected by scintillation. In this sense, carrier-phase residuals in Eq.(5) can be weighted taking into account some of the parameters related with scintillation (e.g. the Signal to Noise Ratio (SNR) or the ROTI for each specific satellite). Notice that, as shown in Fig. 5, the receiver clock offset presents non-negligible fluctuations at high frequency that (as noted in Humphreys et al (2005)) are not completely removed using a high-pass band filter.

Finally, the receiver clock offset estimation is subtracted from the current residual of the $L_{I F}$, thus completing the de-trending of the ionospheric-free combination. The final residual of the $L_{I F}$ is depicted in Fig. 6 . 


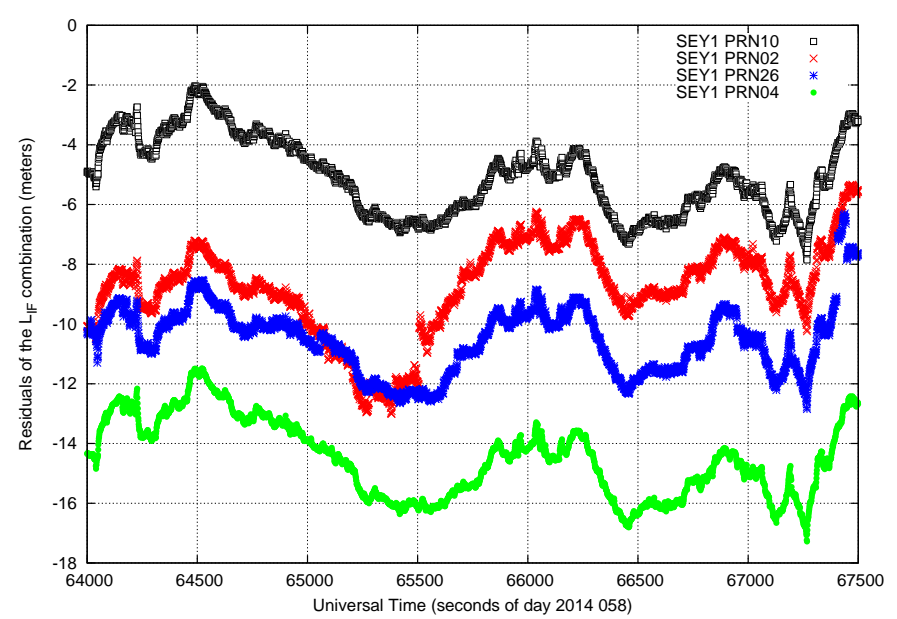

Fig. 4: For IGS receiver SEY1: residual of the detrended ionospheric-free combination of carrier-phase measurements, $L_{I F}$, for GPS satellites: PRN02 (red crosses), PRN04 (green dots), PRN10 (black squares) and PRN26 (blue asterisks).

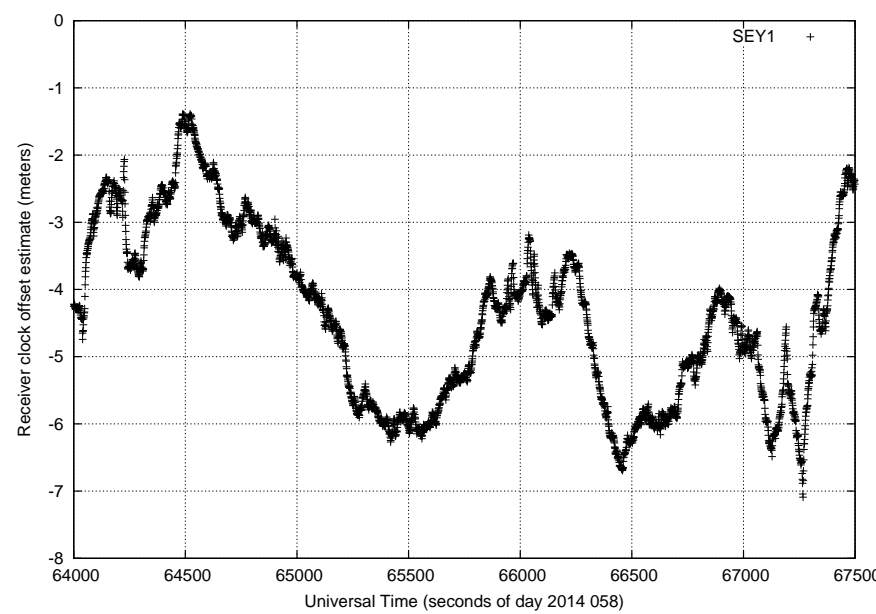

Fig. 5: For IGS receiver SEY1: estimated receiver clock offset for DOY 058, 2014.

Two main effects of scintillation on the low-latitude receiver SEY1 for satellite PRN26 are shown in this figure: (i) an increase of the amount of noise of the $L_{I F}$ combination due to the diffraction of the GNSS signals and (ii) the presence of cycle-slips. Notice that, after applying the proposed methodology, it is possible to distinguish between cycle-slips occurring in $L_{1}(0.484 \mathrm{~m})$ and $L_{2}(0.377 \mathrm{~m})$. As a consequence, if the de-trending is accurate enough, then one could correct these cycleslips at each frequency. For instance, the arc around the second 64500 in Fig. 6, can be corrected by 4 cycles of $L_{2}(1.508 \mathrm{~m})$ for aligning it with the beginning of the interval.

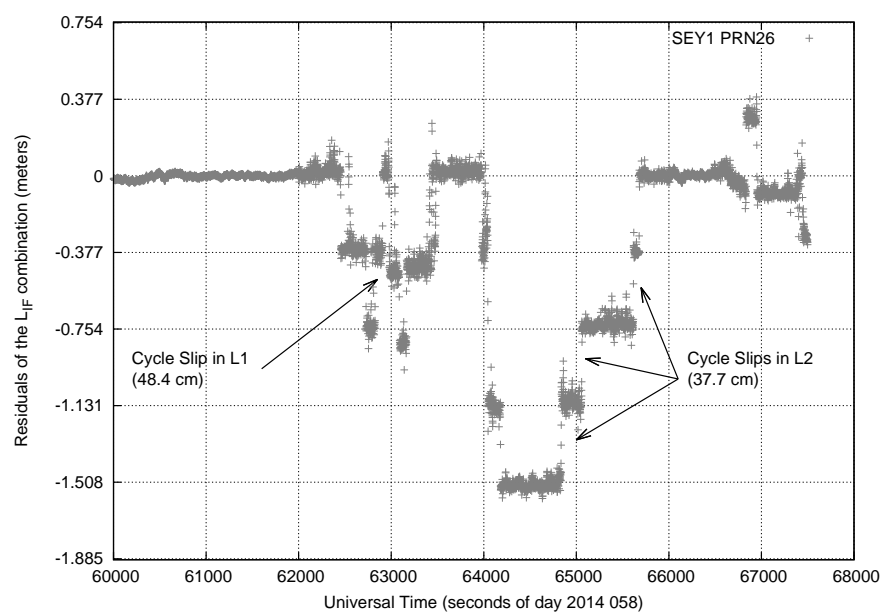

Fig. 6: For IGS receiver SEY1 and PRN26: final residual of $L_{I F}$ once the receiver clock offset estimation (Fig. 5) is subtracted from the de-trended $L_{I F}$ obtained in the previous step (see Fig. 4). The ticks in the Y-axis of $37.7 \mathrm{~cm}$ facilitate the identification of jumps in the L2 frequency, see Eq. (4).

A question arises from the results shown in Fig. 6: are those jumps on the $L_{1}$ or $L_{2}$ carriers inherent to the signal or are they artifacts of the receiver processing? In Xu et al (2015), the authors examined the relationship between the amplitude fading and the phase jumps; they concluded that the phase jumps are different for $L_{1}$ and $L_{5}$ signals and the jumps are not simultaneous with the fading amplitude. From this observation, the authors attributed the phase jumps to the input signal rather than to the receiver. The method presented in the current paper allows for studying long time intervals (the complete satellite-receiver arc of data that can last for hours), which can be helpful to answer the above-mentioned question. Indeed, Fig. 7 depicts the $L_{I F}$ de-trended residuals for the satellite PRN02 together with the $S_{4}$ index for both frequencies $L_{1}$ and $L_{2}$. This particular $S_{4}$ index was derived from the SNR measurements present in the $1-\mathrm{Hz}$ RINEX file. The $S_{4}$ values and the $L_{I F}$ residuals clearly show that there is no scintillation at the beginning and at the end of this continuous arc of data of satellite PRN02, i.e. the perturbation occurs from $64800 \mathrm{~s}$ to $70200 \mathrm{~s}$. Therefore, if the jumps were in the input signal, then the residuals at the beginning and at the end of the de-trended $L_{I F}$ should be aligned. However, this clearly is not the case: there is a bias of $1.5 \mathrm{~m}$, which corresponds to 4 jumps in $L_{2}$ between the beginning (61200 s) and the end (75600 s) of the arc of continuous data. Thus, the conclusion 


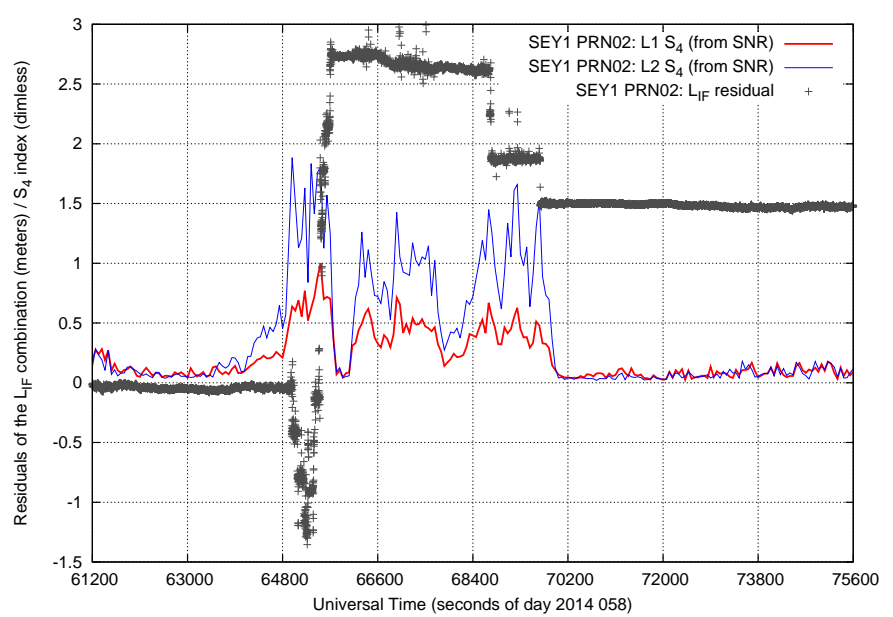

Fig. 7: For IGS receiver SEY1 and PRN02: $L_{I F}$ residual (grey pluses) versus $S_{4}$ index at the $L_{1}$ and $L_{2}$ frequencies (red and blue lines, respectively). The $S_{4}$ indices are derived from the SNR present in the RINEX file.

from this result is that the carrier phase jumps (during a fading of the signal) are produced in the receiver Phase Lock Loop (PLL). In such a way that, under the same scintillation environment, the response of two receivers can be different.

\section{The IF-Sigma indicator}

From the point of view of satellite navigation, the main problem with scintillation is the presence of continuous cycle-slips due to the diffraction of the GNSS signal (Banville et al, 2010). Therefore, if such cycle-slips could be detected and corrected, then it would be possible to use in the navigation filter the carrier-phase measurements from satellites under scintillation. In fact, because of the accurate de-trending introduced in the previous section, one can distinguish a jump in $L_{1}$ from a jump in $L_{2}$. Therefore, these cycle-slips can be detected and corrected. This process is illustrated in the next two figures: Fig. 8 corresponds to the example (SEY1, PRN26) developed in the previous section, and Fig. 9 extends the procedure for all the satellites in view from the IGS receivers in the low and high latitude regions in Table 1 (during the DOY 058, 2014).

Figure 8 and Figure 9 show that, once cycle-slips are corrected, the residuals of the $L_{I F}$ only reach the level of some centimetres. The results for the high latitude receivers (YELL and MCM4) appear to indicate that cycle-slips associated with scintillation are not frequent at those latitudes, even under high ionospheric activity.

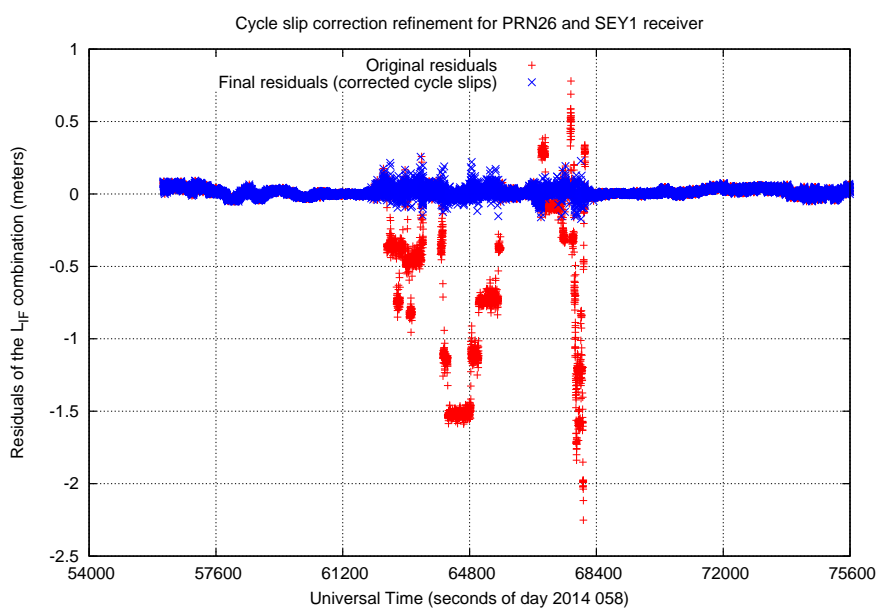

Fig. 8: For IGS receiver SEY1 and PRN26: red pluses, residual of the ionospheric-free combination, $L_{I F}$; blue crosses, residual of $L_{I F}$ after detection and correction of cycle-slips.

Notice that, despite these results correspond to a single day, from the study of additional days, it appears that this is the general situation for high-latitude scintillation that is the presence of cycle-slip is much less frequent than at low-latitude. However, this should be further confirmed by studying longer periods with ionospheric activity.

In fact, the cases with larger residuals experienced in the YELL receiver (for instance, a peak up to one metre in Fig. 9) are associated with errors in the interpolation of satellite orbits or clocks. This association is confirmed in Fig. 10, where the $L_{I F}$ residuals for the satellites PRN01 and PRN28 in view from receivers YELL and USN3 are depicted. Indeed, the residuals exhibit a common pattern, despite the USN3 receiver being located at mid latitude, where no scintillation is expected. Thus, these residuals at the decimetre level (unlike the nominal centimetre-level, as in Fig. 9) are attributable to the interpolation of satellite orbits or clocks and not to scintillation. In the case of PRN01, the problem is a lack of availability in the IGS Final orbit file, whereas in the case of PRN28 the problem is related with fast fluctuations of the satellite clock with a time-scale smaller than the IGS Final clock file. These type of anomalies are not frequent and, as we show, can be detected by using additional receivers in order to see such common patterns.

At this point, considering that the cycle-slips can be identified and corrected, the navigation solution is only affected by the remaining residual of the $L_{I F}$, the 

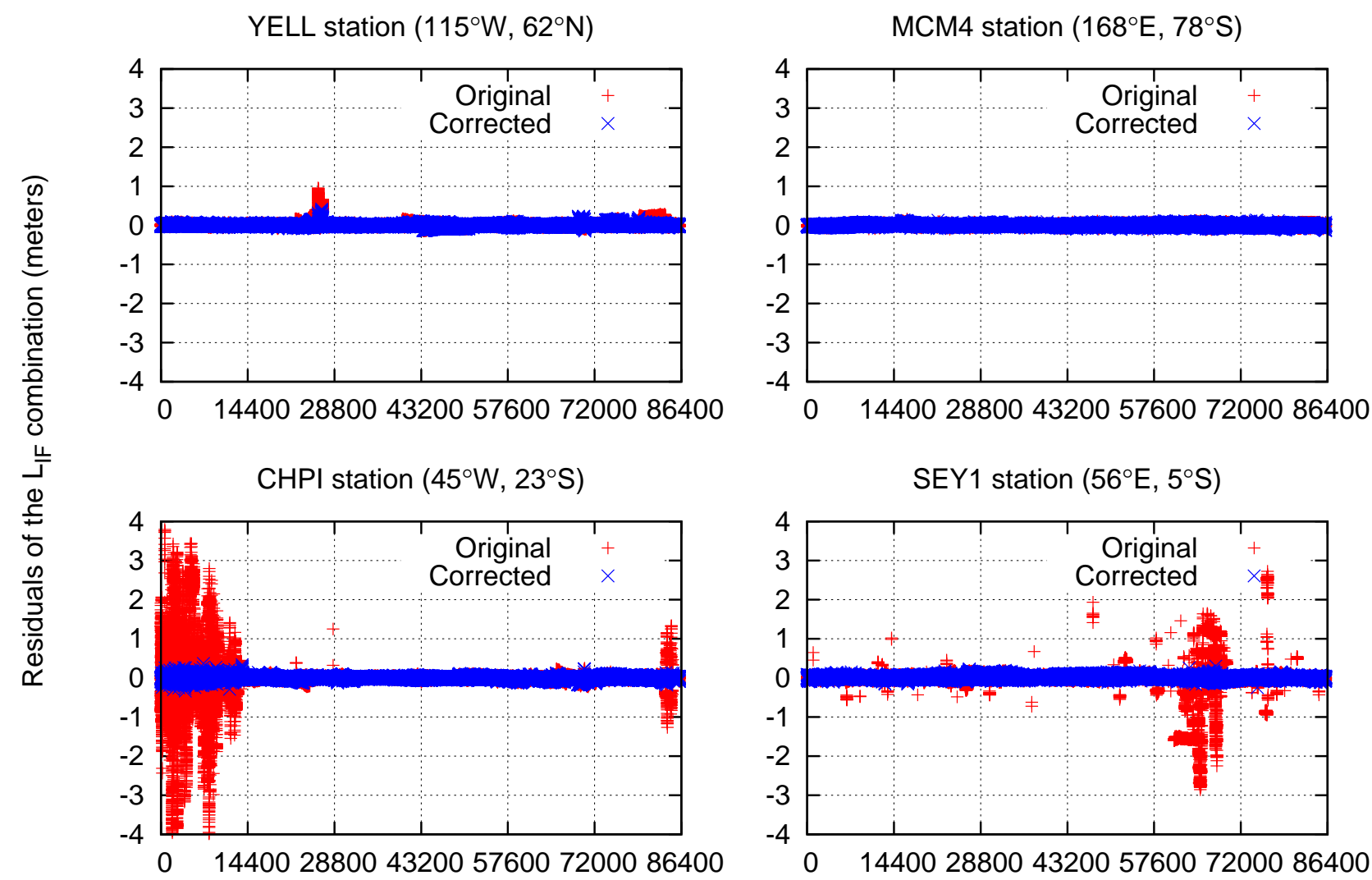

Universal Time (seconds of day 2014 058)

Fig. 9: From the top to bottom row and the left to right column. For high latitude (YELL, MCM4) and low latitude (CHPI, SEY1) receivers: red pluses, residual of the ionospheric-free combination, $L_{I F}$; blue crosses, residual of $L_{I F}$ after detection and correction of cycle-slips. All satellites in view of the receivers are depicted.

diffractive part, which, unlike the refractive part, remains under scintillation (Fig. 8 and Fig. 9). In this sense, the standard deviation of such residuals can be used as a parameter to reflect the degradation in the navigation that any receiver would experience, provided the phase jumps are detected and fixed. Such standard deviation is defined as IF-sigma $\left(\sigma_{I F}\right)$. Figure 11 depicts the $\sigma_{I F}$ parameter (computed over a sliding window of 1 minute) for the example developed in the previous sections. The observed increase of the value of $\sigma_{I F}$ corresponds to the hours associated with the presence of scintillations (observed as rapid fluctuations in the residuals of the $L_{I F}$ combination). Under such circumstances, the degradation of the signal will be translated into the navigation solution.

The results in Fig. 9 support that, with the $\sigma_{I F}$, one can see clearly differences between high and low latitude scintillation. Certainly, depicting $\sigma_{I F}$ in Fig. 12 for the same cases represented in Fig. 2, $\sigma_{I F}$ is significantly smaller for the high latitude receivers (YELL, MCM4)

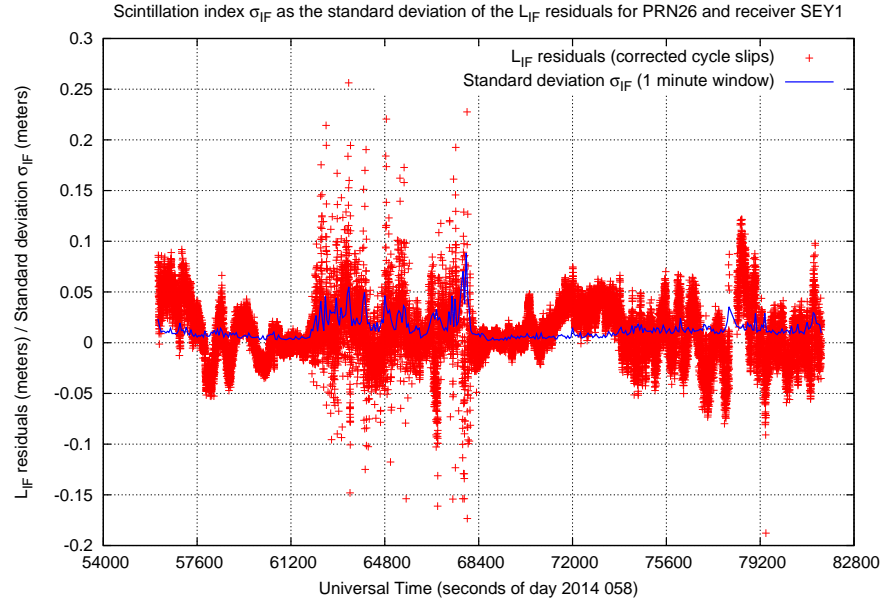

Fig. 11: For IGS receiver SEY1 and PRN26: red pluses, residual of the de-trended and receiver-clock free ionospheric-free combination, $L_{I F}$; blue line, corresponding $\sigma_{I F}$. 

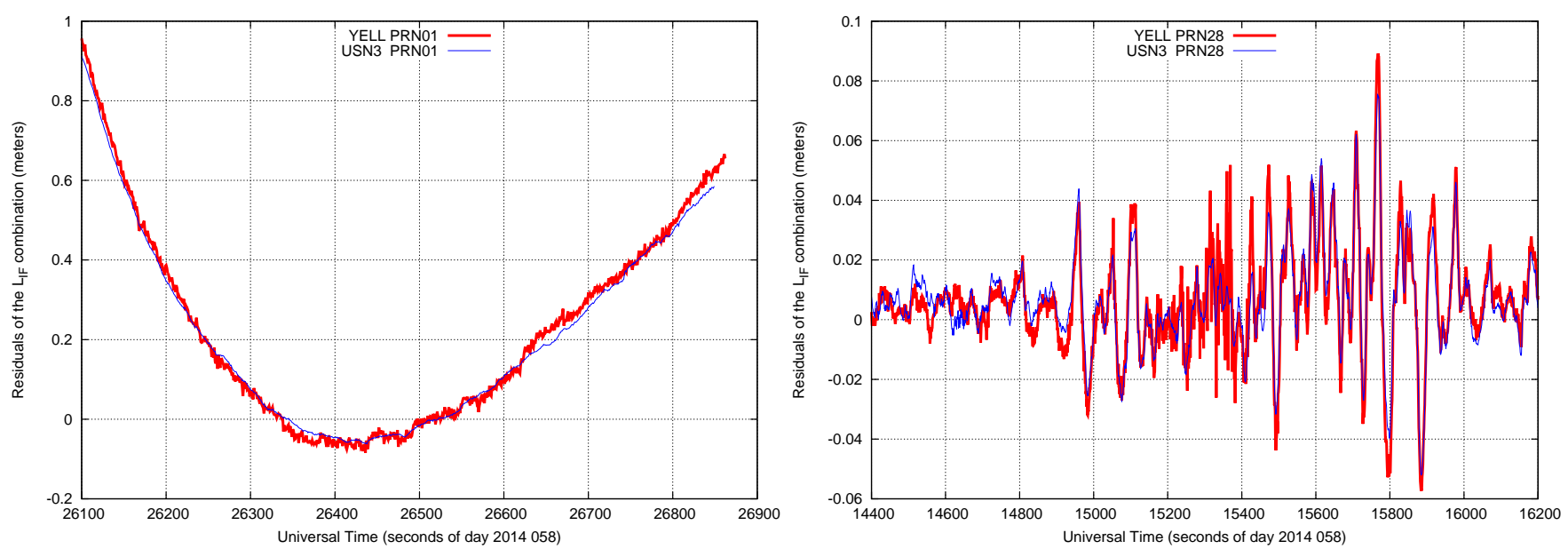

Fig. 10: $L_{I F}$ residuals for receivers YELL (red) and USN3 (blue). The signal de-trending can be affected by holes in the SP3 precise orbit file (left-hand plot, PRN01) or by non-linear behaviour of the satellite clock (right-hand plot, PRN28).

than for the low latitude ones (CHPI, SEY1). Indeed, the values of $\sigma_{I F}$, for high latitude receivers are of 1 or 2 centimetres, which are comparable to the degradation of the $\sigma_{I F}$ with the elevation angle, due to miss modelling in the $L_{I F}$ (as can be seen at the beginning or the end of arcs in Fig. 12); thus, in general if the $\sigma_{I F}$ with such small values, a high degradation on the navigation solution should not be expected in these regions when one uses the classical PPP technique (i.e., using the $L_{I F}$ combination). This agrees, for example, with Jacobsen and Andalsvik (2016), where in spite of a slight increase of the navigation error with the ionospheric activity, it is maintained at the level of few centimetres.

\section{IF-sigma versus other scintillation indices}

In this section, the newly defined $\sigma_{I F}$ index is compared to the most commonly used scintillation indices $\left(\sigma_{\varphi}, S_{4}\right.$ and ROTI).

The newly introduced $\sigma_{I F}$ is, by definition, directly affected by the mis-modelling of the GNSS signal. This mis-modelling is usually at the level of 1 centimetre (in Root Mean Square (RMS) and smaller in 1-sigma value) and increases at low elevation angles. The latter can be mitigated with an elevation cut-off angle (approximately 20 degrees) or by means of an obliquity factor. Taking this factor into account, $\sigma_{I F}$ is able to detect scintillation when its effect on the $L_{I F}$ combination is greater than 1 centimetre. From the conversion values in Eq. (4), this situation implies a detection capability of the $\sigma_{I F}$ indicator of approximately 0.1 radians.

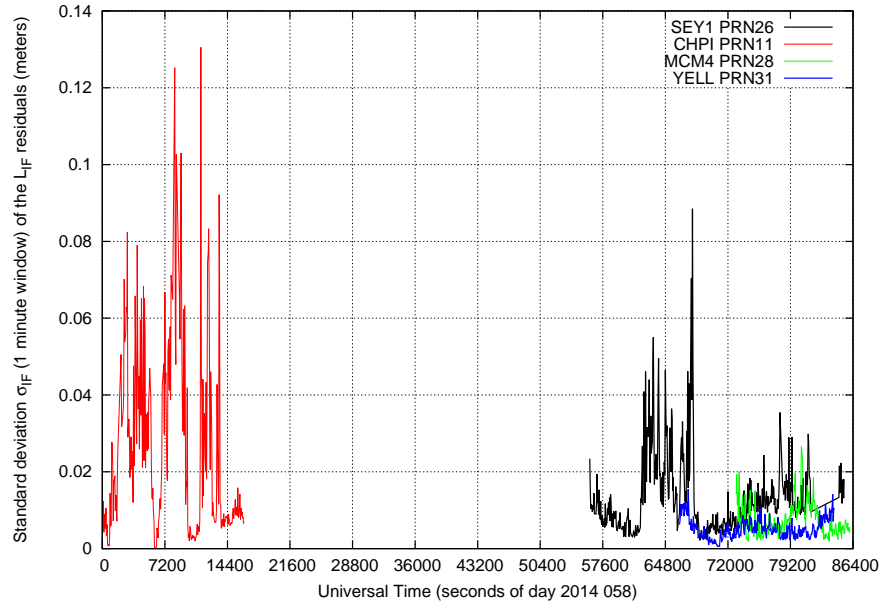

Fig. 12: Standard deviation $\sigma_{I F}$ of the $L_{I F}$ residuals for DOY 058, 2014 at different IGS stations placed at high latitude: MCM4 (green) and YELL (blue); and low latitude: SEY1 (black), CHPI (red) for some particular satellites (PRN26, PRN11, PRN28 and PRN31).

Two additional aspects shall be considered when comparing the $\sigma_{I F}$ with the $\sigma_{\varphi}$ values:

(i) For the $\sigma_{\varphi}$ computation, it is not clear how the receiver de-trending algorithms function when a cycleslip occurs (notice that, as reported in $\mathrm{Xu}$ and Morton (2015), these jumps can last for several tens of milliseconds). As a result, different receivers with different PLLs or de-trending algorithms present different $\sigma_{\varphi}$ values (Humphreys et al, 2005).

(ii) Because $L_{I F}$ is a combination of $L_{1}$ and $L_{2}$, a $\sigma_{I F}$ can be intended to be derived (let us name it $\sigma^{*}$ ) from the respectively $\sigma_{\varphi}$ associated with $L_{1}$ and $L_{2}$ (or 
$\left.L_{5}\right)$ using the conversion factors of Eq. (4). However, this resulting $\sigma^{*}$ depends on the correlation between the scintillation effects on $L_{1}$ and $L_{2}$. For example, if the effect in both frequencies were totally uncorrelated, then the value of $\sigma^{*}$ would satisfy the following relationship:

$\sigma^{* 2}=\alpha^{2} \cdot \sigma_{1}^{2}+\beta^{2} \cdot \sigma_{2}^{2}$

where $\sigma_{1}$ and $\sigma_{2}$ correspond to the $\sigma_{\varphi}$ values on $L_{1}$ and $L_{2}$, and $\alpha$ and $\beta$ are the parameters defined in Eq. (4).

However, in the case of having some correlation in the scintillation effect on both frequencies, Equation Eq. (6) is different. For example, if the scintillation were only refractive, then the ionospheric delay in both frequencies would be completely correlated. Under such circumstances, the relationship between $\sigma_{1}$ and $\sigma_{2}$ is (Rino, 1979):

$\frac{\sigma_{1}}{\sigma_{2}}=\frac{f_{1}}{f_{2}}=\frac{\beta}{\alpha}$

where $f_{1}$ and $f_{2}$ denote the $L_{1}$ and $L_{2}$ frequencies. In this case, scintillation would not affect the $L_{I F}$ combination and $\sigma_{I F}$ should be quite small (reflecting only a small mis-modelling of $L_{I F}$ ).

Considering the previous comments, the comparison of $\sigma_{I F}$ with the standard $\sigma_{\varphi}$ measurements presents some difficulties related with the correlation of the scintillation effect on both frequencies. However, as a starting point based on the cases shown in the previous sections, we can assume that, in high latitude regions, the effect of scintillation at both frequencies is quite correlated (Wang and Morton, 2015), whereas in low latitude regions, the effect of scintillation at both frequencies is quite uncorrelated (see also $\mathrm{Xu}$ and Morton, 2015).

For example, in Fig. 13, $\sigma^{*}$ is depicted, derived from $\sigma_{1}$ and $\sigma_{5}$ (assuming uncorrelated values of $\sigma_{\varphi}$ ) versus $\sigma_{I F}$ for an ISMR located at the Jicamarca Radio Observatory (JRO) in Peru $\left(77^{\circ} \mathrm{W}, 12^{\circ} \mathrm{S}\right)$. During DOY 298 of 2014 , the ionospheric activity in this region presented the highest values for the whole year of 2014. The values of $\sigma^{*}$ and $\sigma_{I F}$ (from $L_{1}$ and $L_{5}$ ) are depicted for the GPS satellite PRN27. In this comparison, a reasonable match is found between their values under the assumption that the scintillation effect in both frequencies is uncorrelated. Moreover, a good correlation is found between the $S_{4}$ and the $\sigma_{I F}$, as shown in the bottom panel of Fig. 13.

Taking into account the results of Sect. 4 and 5 , we can conclude that, at high latitudes (small values of $\left.\sigma_{I F}\right)$, scintillation is mostly linked to the refractive part of the ionospheric delay. In other words, phase fluctuations associated to scintillation in $L_{1}$ and $L_{2}$ are quite correlated (both are proportional to $f^{-2}$ ). This agrees with the moderate values of $S_{4}$ seen at these regions, (see, for instance Béniguel et al, 2009). This phenomenon would not be the case for low latitude regions, where, due to the relevance of the signal diffraction, scintillations at both frequencies appear to be more uncorrelated, thus producing, apart from cycleslips, larger values of $\sigma_{I F}$. Moreover, the amplitude of the carrier phase will be affected, resulting in higher values of $S_{4}$. One comparison that highlights these conclusions is shown in Fig. 14. For high latitude (YELL, MCM4) and low latitude (CHPI, SEY1) receivers, $\sigma_{I F}$ is depicted in the top panel; $S_{4}$ (derived from the SNR included in the RINEX file) is shown in the central panel, and, in the bottom panel, the ROTI is shown after correcting for cycle-slips. In summary, as reported in previous works (see, for example Steenburgh et al, 2008), the $S_{4}$ parameter is typically higher for low latitude measurements than for high latitude ones, as confirmed from the results shown in Fig. 14. This observation reinforces the assumption that $\sigma_{I F}$ and $S_{4}$ are both related to diffractive processes that affect the signal amplitude.

In addition, a noticeable result from Fig. 14 is the change of the ROTI values for the low latitude receivers when they are compared with those in Fig. 2. Indeed, the ROTI values for high latitude receivers (YELL, MCM4) remain the same (because the STEC values are not affected by cycle-slips), whereas this is not the case for the low latitude receivers (CHPI or SEY1): the ROTI values are reduced significantly (almost by $50 \%$ ) when STECs are corrected from cycle-slips. Consequently, for low latitudes, higher ROTI values can be associated with cycle-slips not correctly detected rather than with ionospheric scintillation.

Figure 15 depicts an example of situation where the ROTI values can be incorrectly computed as a result of a miss-detection of cycle-slips. The raw STEC (depicted with red squares) is affected by 5 cycle-slips as it can be clearly seen in the residuals of the $L_{I F}$ combination (shown with solid black line). The STEC, once these cycle slips are corrected, is shown with blue circles. The bottom plot illustrates that the ROTI computed with the STEC and the cycle-slips correctly detected presents different values than those from the raw STEC from the RINEX files. Notice that usual cycleslip detectors based in the continuity of the $L_{G F}$ (look- 

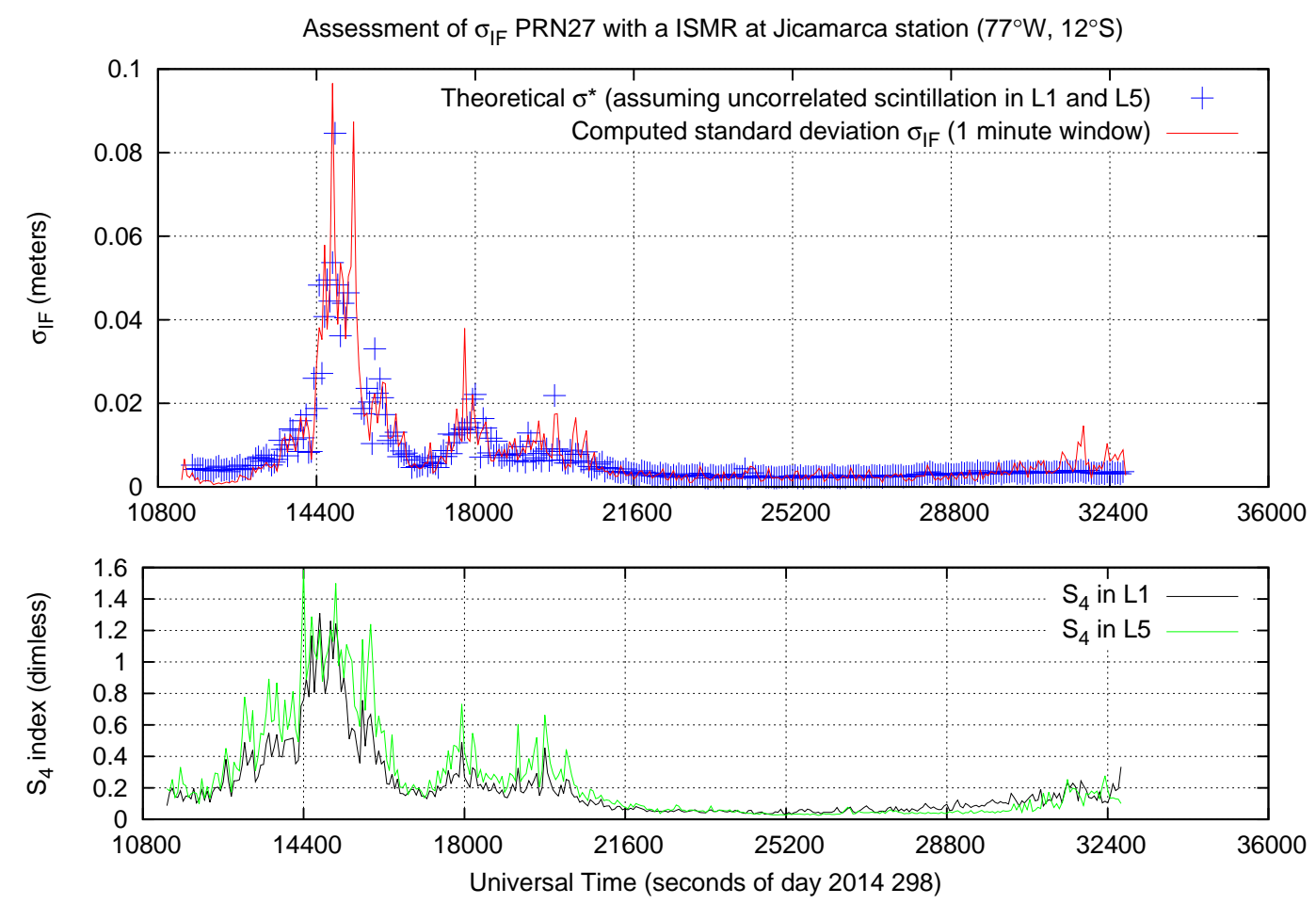

Fig. 13: For the ISMR receiver JRO and PRN27: Top panel depicts the $\sigma^{*}$ (blue pluses) and $\sigma_{I F}$ (red line) values during DOY 298 of 2014. Bottom panel depicts the $S_{4}$ for L1 and L5 frequencies (black and green lines, respectively).
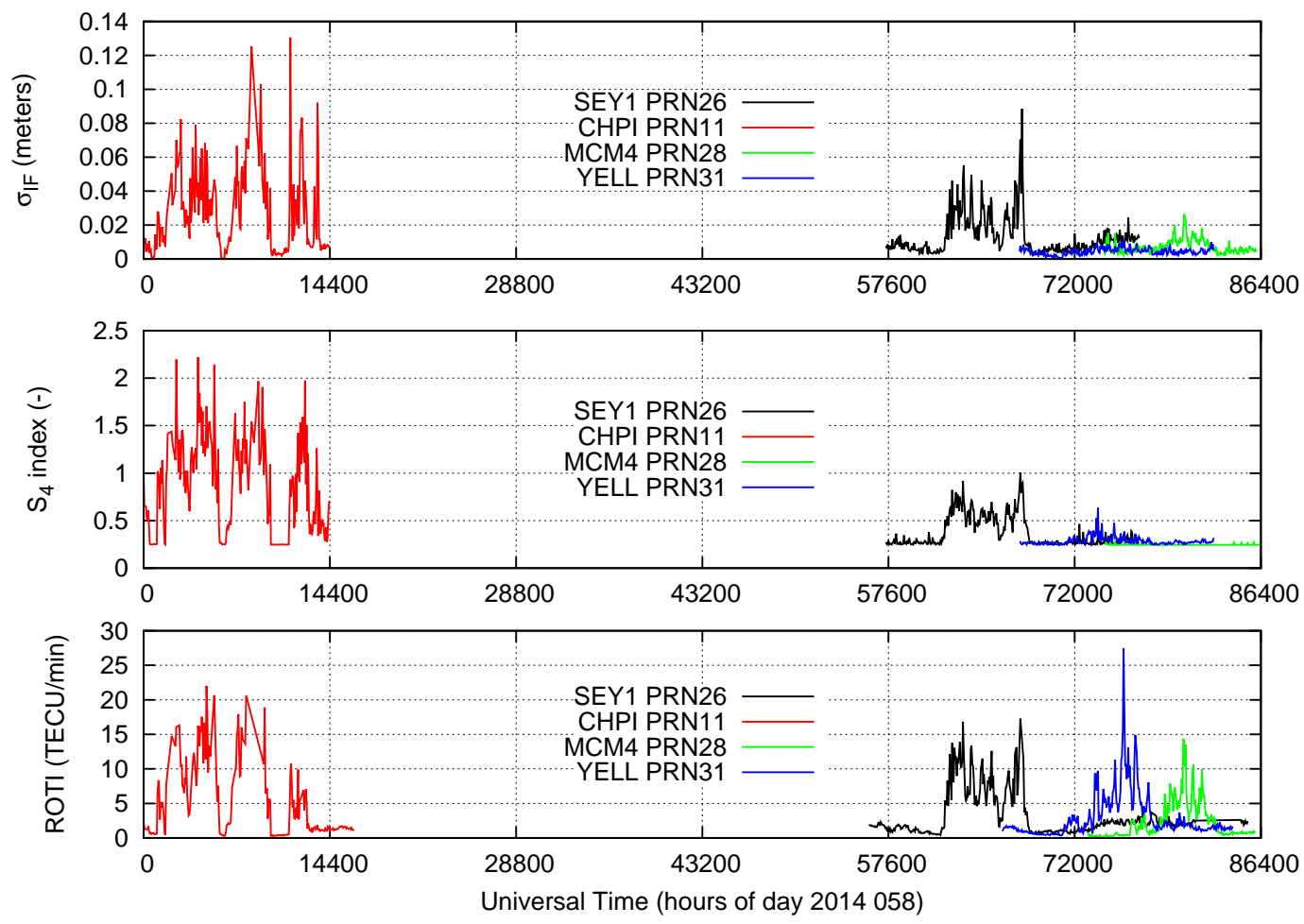

Fig. 14: Top panel, $\sigma_{I F}$; central panel, $S_{4}$; bottom panel, ROTI after correcting for cycle-slips. Results correspond to DOY 058, 2014 at different IGS stations placed at high latitude: MCM4 (green) and YELL (blue); and low latitude: SEY1 (black), CHPI (red) for some particular satellites (PRN26, PRN11, PRN28 and PRN31). 


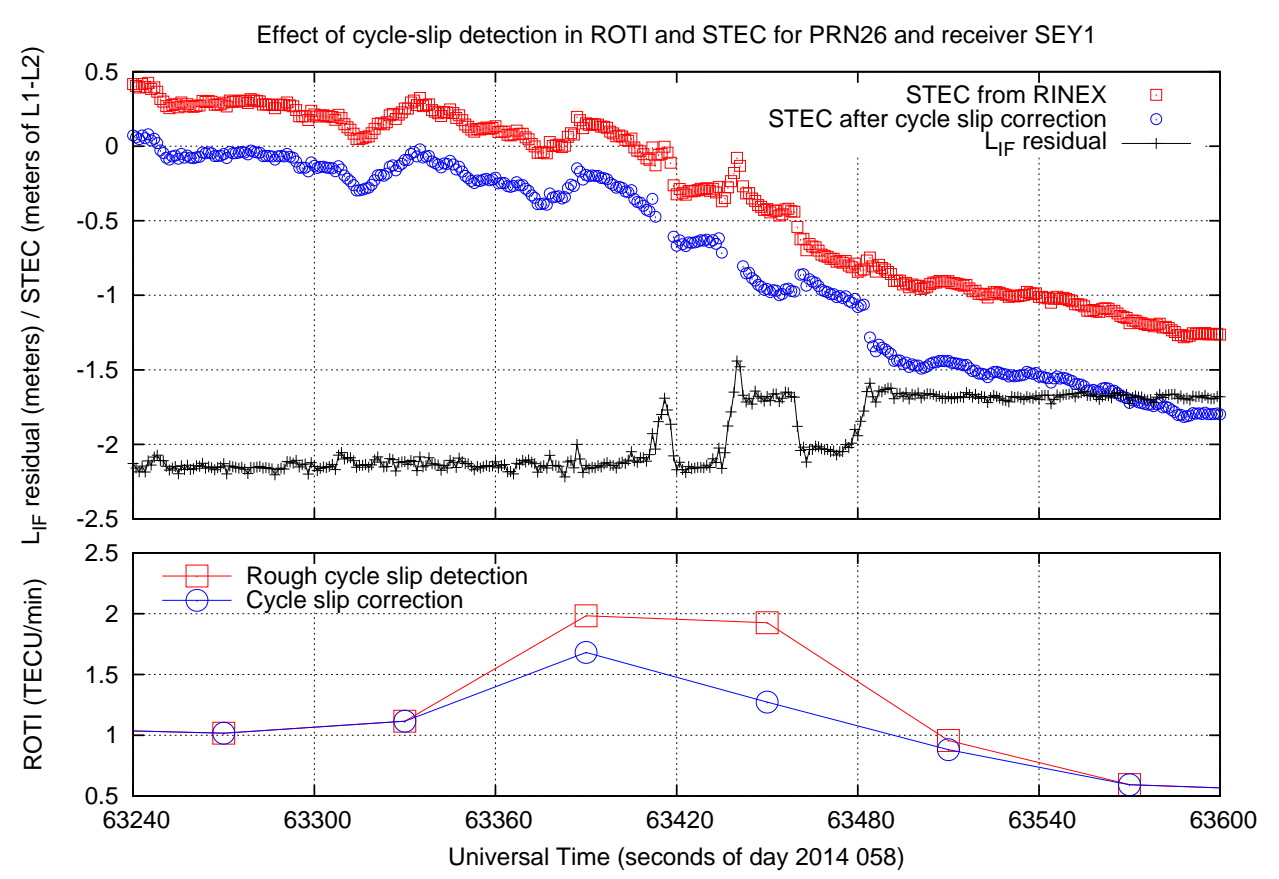

Fig. 15: For IGS receiver SEY1 and PRN26: Top panel depicts with red squares the STEC from RINEX without cycle-slip detection; blue circles, STEC during different continuous arcs of data after correction of cycle-slips; black line with pluses, residual of the de-trended and receiver-clock free ionospheric-free combination, $L_{I F}$, before cycle slip detection and repair. The bottom plot depicts the corresponding ROTI values with the rough cycle-slip detection (red squares) and the final cycle-slip correction (blue circles).

ing for abrupt changes) would not detect most of these cycle-slips (because they are drifts), in particular, the last one around second 63480 .

Finally, from the comparison between ROTI and $\sigma_{I F}$ depicted in Fig. 14, one can conclude that, for high latitudes, high ROTI values do not always imply high values of $\sigma_{I F}$. This finding is important from the point of view of navigation. Indeed, if the navigation solution is obtained using the $L_{I F}$ combination, the accuracy of the solution will depend just on the $\sigma_{I F}$, regardless of the ROTI value. In this sense, high ROTI values (with small $\sigma_{I F}$ values) indicate large spatial or temporal gradients in the ionosphere (with a refractive origin that cancels out when the ionospheric-free combination is built). This would only affect navigation in the case of differential techniques (where the user applies external ionospheric corrections computed from the measurements from other receivers).

\section{Conclusions}

The present paper introduced a method capable of detecting scintillation on the GNSS signals based on its effect in the ionospheric-free combination of carrier phase measurements collected by geodetic receivers. As it known, this combination is the standard one used in PPP. In this manner, the effect of scintillation on this combination is translated directly into high-accuracy navigation (if many satellites are affected).

One of the main advantages of this novel approach is that it can be applied even with data collected at a $1-\mathrm{Hz}$ sampling rate, which corresponds to the data collected by hundreds of deployed GNSS receivers throughout the world. Therefore, this technique allows for conducting studies over long periods of time and on a planetary scale, which enable new opportunities for scintillation investigations. Indeed, the novel approach overcomes the limitations of the current techniques caused by: (i) the reduced number of ISMRs available and (ii) their data are only provided for short periods of time.

It has been shown that, by means of a rough cycleslip detector combined with accurate modelling of the ionospheric-free combination (including all well-known effects, such as satellite clock offsets and receiver clock offset), it is possible to isolate the mis-modelling in the residual of this combination. This final residual clearly shows the two main effects of scintillation: the presence 
of cycle-slips and an increased noise in the ionosphericfree combination due to the diffraction of the signal.

By considering the effect of single jumps in the individual frequencies, the proposed method is able to isolate, over the combined signal, the frequency experiencing the cycle-slip. Because of this identification and the subsequent repair of cycle-slips, the navigation errors are only affected by the remaining residual of the ionospheric-free combination. The standard deviation of such residuals constitutes a new ionospheric scintillation index that reflects the degradation in the user navigation. This new ionospheric scintillation index, derived from data at $1 \mathrm{~Hz}$ and named $\sigma_{I F}$, was compared with the standard scintillation indices $\left(\sigma_{\varphi}\right.$ and $\left.S_{4}\right)$ provided by an ISMR receiver working at $50 \mathrm{~Hz}$. These comparisons have demonstrated that $\sigma_{I F}$ accounts for essentially the same diffractive effect on the GNSS signal as $S_{4}$ does. Moreover, since $\sigma_{I F}$ is computed from the $L_{I F}$, its value relates the degradation of precise navigation with the scintillation.

Finally, using the methodology developed in this work, the ROTI index is found to be a rough indicator of the ionospheric activity. On the one hand, because ROTI is based on the geometry-free combination, it does not distinguish diffractive effects (large $S_{4}$ and $\sigma_{I F}$ values) from refractive effects (moderate $S_{4}$ and $\sigma_{I F}$ values). On the other hand, if the cycle-slips associated with scintillation are not corrected, then the ROTI value will be overestimated, being large values of ROTI linked to the receiver internal software processes rather than to actual ionospheric activity.

Acknowledgements The authors acknowledge the use of data from the International GNSS Service and from the Joint Research Centre.

\section{References}

Aarons J (1982) Global morphology of ionospheric scintillations. Proceedings of the IEEE 70(4):360-378, DOI 10.1109/PROC.1982.12314, URL http://dx. doi .org/10.1109/PROC.1982.12314

Aarons J (1993) The longitudinal morphology of equatorial f-layer irregularities relevant to their occurrence. Space Science Reviews 63(3):209-243, DOI 10. 1007/BF00750769, URL http://dx.doi.org/10. 1007/BF00750769

Banville S, Langley RB, Saito S, Yoshihara T (2010) Handling cycle slips in gps data during ionospheric plasma bubble events. Radio Science 45(6):1-14, DOI 10.1029/2010RS004415, URL http://dx.doi. org/10.1029/2010RS004415

Basu S, Basu S (1989) Scintillation technique for probing ionospheric irregularities. In: Liu, CH (ed) World Ionospheric and Termospheric Studies (WITS) Handbook, Vol. 2, Urbana, Illinois (USA), pp 128-136

Béniguel Y, Romano V, Alfonsi L, Aquino M, Bourdillon A, Cannon P, Franceschi GD, Dubey S, Forte B, Gherm V, Jakowski N, Materassi M, Noack T, Pozoga M, Rogers N, Spalla P, Strangeways H, Warrington EM, Wernik A, Wilken V, Zernov N (2009) Ionospheric scintillation monitoring and modelling. Annals of Geophysics 52(3-4), URL http: //www . annalsofgeophysics . eu/index . php/annals/article/view/4595

Beutler G, Rothacher M, Schaer S, Springer T, Kouba J, Neilan R (1999) The International GPS Service (IGS): An interdisciplinary service in support of Earth sciences. Advances in Space Research 23(4):631-653, DOI 10.1016/ S0273-1177(99)00160-X, URL http://dx.doi.org/ 10.1016/S0273-1177 (99)00160-X

Blewitt G (1990) An automatic editing algorithm for gps data. Geophysical Research Letters 17(3):199 202, DOI 10.1029/GL017i003p00199, URL http:// dx.doi.org/10.1029/GL017i003p00199

Briggs B, Parkin I (1963) On the variation of radio star and satellite scintillations with zenith angle. Journal of Atmospheric and Terrestrial Physics 25(6):339 - 366, DOI 10.1016/0021-9169(63)90150-8, URL http://dx.doi .org/10.1016/0021-9169(63) 90150-8

Carrano C, Groves KM (2007) TEC Gradients and Fluctuations at Low Latitudes Measured with High Data Rate GPS Receivers. In: Proceedings of ION 2007, Cambridge MA (USA), pp 156163, URL https://www.ion.org/publications/ abstract. $c$ fm?articleID $=7243$

Carrano C, Groves KM, McNeil WJ, Doherty PH (2013) Direct Measurement of the Residual in the Ionosphere-Free Linear Combination during Scintillation. In: Proceedings of ION ITM 2013, San Diego, CA (USA), pp 585-596, URL http://www.ion.org/ publications/abstract. cfm?articleID $=10893$

Cervera MA, Thomas RM (2006) Latitudinal and temporal variation of equatorial ionospheric irregularities determined from gps scintillation observations. Annales Geophysicae 24(12):3329-3341, 
DOI 10.5194/angeo-24-3329-2006, URL http:// www . ann-geophys . net/24/3329/2006/

Crawford F (1968) Berkeley Physics Course, Vol. 3, Waves. McGraw-Hill,, New York, USA

Forte B (2005) Optimum detrending of raw GPS data for scintillation measurements at auroral latitudes. Journal of Atmospheric and SolarTerrestrial Physics 67(12):1100 - 1109, DOI http://dx.doi.org/10.1016/j.jastp.2005.01.011,

URL http://www.sciencedirect.com/science/ article/pii/S136468260500091X

Humphreys T, Psiaki M, Kintner PJ, BM L (2005) GPS Carrier Tracking Loop Performance in the Presence of Ionospheric Scintillation. In: Proceedings of ION GNSS 2005, Long Beach, CA (USA), pp 156-167, URL http://www.ion.org/ publications/abstract. $\mathrm{cfm}$ ? articleID $=6206$

International GNSS Service Products (2014) URL http: //www.igs.org/products/data

Jacobsen K (2014) The impact of different sampling rates and calculation time intervals on ROTI values. J Space Weather Space Clim 4:A33, DOI 10.1051/ swsc/2014031, URL http://dx.doi.org/10.1051/ swsc/2014031

Jacobsen K, Andalsvik Y (2016) Overview of the 2015 St. Patricks day storm and its consequences for RTK and PPP positioning in Norway. J Space Weather Space Clim 6:A9, DOI 10.1051/swsc/2016004, URL http://dx.doi.org/10.1051/swsc/2016004

Kintner P, Humphreys T, J H (2009) How to survive the next solar maximum. Inside GNSS (July/August 2009) 4(4):22-30, URL www. insidegnss . com/auto/ julyaug09-kintner.pdf

Paznukhov VV, Carrano CS, Doherty PH, Groves KM, Caton RG, Valladares CE, Seemala GK, Bridgwood CT, Adeniyi J, Amaeshi LLN, Damtie B, D’Ujanga Mutonyi F, Ndeda JOH, Baki P, Obrou OK, Okere B, Tsidu GM (2012) Equatorial plasma bubbles and l-band scintillations in africa during solar minimum. Annales Geophysicae 30(4):675-682, DOI 10.5194/angeo-30-675-2012, URL http://www . ann-geophys . net/30/675/2012/

Pi X, Mannucci AJ, Lindqwister UJ, Ho CM (1997) Monitoring of global ionospheric irregularities using the Worldwide GPS Network. Geophysical Research Letters 24(18):2283-2286, DOI 10.1029/97GL02273, URL http://dx.doi.org/10.1029/97GL02273

Pi X, Iijima BA, Lu W (2014) Effects of Ionospheric Scintillation on GNSS-Based Position- ing. In: Proceedings of ION GNSS+ 2014, Tampa, Florida (USA), pp 1090-1100, URL http://www.ion.org/publications/abstract . cfm?jp=p\&articleID $=12226$

Rino CL (1979) A power law phase screen model for ionospheric scintillation: 1. weak scatter. Radio Science 14(6):1135-1145, DOI 10.1029/ RS014i006p01135, URL http://dx.doi.org/10. 1029/RS014i006p01135

Sanz J, Juan J, Hernández-Pajares M (2013) GNSS Data Processing, Vol. I: Fundamentals and Algorithms. ESA Communications, ESTEC TM-23/1, Noordwijk, the Netherlands, URL http://www.navipedia.net/GNSS_Book/ESA_ GNSS-Book_TM-23_Vol_I.pdf

Sanz J, Juan J, González-Casado G, Prieto-Cerdeira R, Schlueter S, Orús R (2014) Novel Ionospheric Activity Indicator Specifically Tailored for GNSS Users. In: Proceedings of ION GNSS+ 2014, Tampa, Florida (USA), pp 1173-1182, URL http://www .ion.org/publications/abstract . cfm?jp=p\&articleID=12269

Steenburgh RA, Smithtro CG, Groves KM (2008) Ionospheric scintillation effects on single frequency gps. Space Weather 6(4):n/a-n/a, DOI 10.1029/2007SW000340, URL http://dx.doi.org/ 10.1029/2007SW000340

Van Dierendonck A, Arbesser-Ratsburg B (2004) Measuring Ionospheric Scinitllation in the Equatorial Region over Africa, Including Measurements from SBAS Geostationary Satellite Signals. In: Proceedings of ION GNSS 2005, Long Beach, CA (USA), pp 316 - 324, URL http://www.ion.org/publications/ abstract. $c f m$ ?articleID $=5710$

Wang J, Morton Y (2015) Spaced Receiver Array for Ionospheric Irregularity Drift Velocity Estimation Using Multi-band GNSS Signals. In: Proceedings of ION GNSS+ 2015, Tampa, Florida (USA), pp 34493458, URL http://www.ion.org/publications/ abstract. $c f m$ ? jp=p\&articleID=12885

$\mathrm{Xu}$ D, Morton Y (2015) GPS carrier parameters characterization during strong equatorial ionospheric scintillation. In: Proceedings of ION ITM 2015, Dana Point, CA (USA), pp 521 - 529, URL http://www.ion.org/publications/ abstract. $c f m$ ? jp=p\&articleID $=12677$

Xu D, Morton Y, Akos D, Walter T (2015) GPS Multi-Frequency Carrier Phase Characterization During Strong Equatorial Ionospheric Scintil- 
lation. In: Proceedings of ION GNSS+ 2015, Tampa, Florida (USA), pp 3787-3796, URL http://www .ion.org/publications/abstract . cfm? jp=p\&articleID $=13011$

Yeh C, Chao-Han L (1982) Radio wave scintillations in the ionosphere. Proceedings of the IEEE 70(4):324360, DOI 10.1109/PROC.1982.12313, URL http:// dx.doi.org/10.1109/PROC.1982.12313

Zumberge JF, Heflin MB, Jefferson DC, Watkins MM, Webb FH (1997) Precise Point Positioning for the efficient and robust analysis of GPS data from large networks. Journal of Geophysical Research: Solid Earth 102(B3):5005-5017, DOI 10.1029/96JB03860, URL http://dx.doi.org/10.1029/96JB03860 